\title{
Coalesced Gas Turbine and Power System Modeling and Simulation using Modelica
}

\author{
Miguel Aguilera $^{1} \quad$ Luigi Vanfretti $^{2} \quad$ Tetiana Bogodorova $^{3} \quad$ Francisco Gómez $^{4}$ \\ ${ }^{1}$ Instituto Costarricense de Electricidad (ICE), Costa Rica, maguilerach@ice.go. cr \\ ${ }^{2}$ Electrical, Computer, and Systems Engineering, Rensselaer Polytechnic Institute (RPI), USA, vanfrl@ rpi . edu \\ ${ }^{3}$ Ukrainian Catholic University, Faculty of Applied Sciences, Lviv, Ukraine, tetiana.bogodorova@gmail. com \\ ${ }^{4}$ KTH Royal Institute of Technology, Stockholm, Sweden, fragomekth. se
}

\begin{abstract}
This work reports how the multi-domain physical modeling and simulation Modelica language has been employed to create a benchmark power grid and gas turbine model within the ITEA3 OpenCPS project. The modeling approach is not only shown to be useful to test the functionalities of the OpenCPS toolchains, but it also could give rise to potential applications in power system domain studies where the widely-accepted turbinegovernor models are not rich enough to represent the multi-domain system dynamics.
\end{abstract}

Keywords: Gas turbine modeling, Modelica, Multidomain modeling and simulation, Power systems, OpenIPSL, ThermoPower

\section{Introduction}

\subsection{Motivation}

Variable energy resources, like wind and solar power, require a special attention due to the challenges that its intermittent nature poses to the power grid operation. To safely integrate these energy sources to power systems, acceptable levels of reliability and security and affordable prices are required (Carnegie Mellon University, 2013).

The operational flexibility of gas power plants makes them a good complement to variable renewable sources. It is very likely that policies will promote the increase of gas power, at least in the next decade, especially because they produce less emissions than coal power plants (IEA, 2016).

The variability of wind and solar power can be expressed as slow or fast fluctuations. Both, the less environment-friendly coal power plants and, combined cycle gas plants can be used to compensate slow power intermittency. On the other hand, power increase/reduction required to deal with fast power fluctuations can be achieved by means of fast response sources like gas natural turbines (Carnegie Mellon University, 2013).

Resilient operation of power systems with high penetration of variable energy resources (VERs) depends, among other factors, on more trustable forecasts and accurate models that can be tailored to the several kinds of power system simulations and analysis. Existing gas turbine models, such as GGOV1, IEEE (De Mello \& Ahner, 1994) and Rowen (Rowen, 1983, 1992), have different levels of complexity and accuracy. Simplicity was a desired property for the first proposed models, primarily due to computer power and turbine modeling data availability limitations of the time when they were proposed, the 1980s or early 1990s (De Mello \& Ahner, 1994; Hannett \& Khan, 1993). Such was the case of the GAST model which was widely used in the United States, but was demonstrated to be inaccurate and thus replaced by the somewhat more complex GGOV1 model (Pereira, Undrill, Kosterev, Davies, \& Patterson, 2003). The widely-accepted models GGOV1, IEEE and Rowen do not employ a detailed physical representation of the gas turbine dynamics; instead, they model dynamics using abstractions in the form of logic and transfer functions which results in loss of information of non-linear physical dynamics. In fact, it has been recently shown (Yee, Milanovic, \& Hughes, 2008) that more detailed models are required to include the grid frequency dependency behavior of gas turbines with the aim of undertaking power system stability studies when the turbines are exposed to abnormal system frequency behavior (e.g. black start, islanded operation, etc.). On the other hand, the correctness of the more complex physical models of gas turbines relies on the availability of turbine modeling data from the manufacturers, who create and then share such models with turbine owners, but are rarely available to most grid analysts due to IP concerns (Yee et al., 2008).

The CEN-CENELEC-ETSI Group recommends the use of the IEC CIM (Common Information Model) for information exchange, which has been mandated at the EU level (CEN-CENELEC-ETSI Smart Grid Coordination Group, 2012). The information exchange required to meet the needs of coordination of transmission system operators (TSOs) operation under any conditions, should comprise both steady-state and dynamic models that can be used for power system simulation. Although the CIM is currently addressing the requirement of dynamic information exchange

The work of F. Gómez was supported in part by the European ITEA3 openCPS project.

The work of L. Vanfretti was supported in part by the Engineering Research Center Program of the National Science Foundation and the Department of Energy under Award EEC-1041877 and in part by the CURENT Industry Partnership Program. 
through IEC-61970-302 and IEC 61970-457, this still might lead to the exchange of ambiguous models as it is explained in a previous work (Vanfretti, Li, Bogodorova, \& Panciatici, 2013). The use of Modelica for dynamic model exchange may help in addressing the challenges as described next.

\subsection{Previous Work}

Specialized tools that allow the modeling and simulation of multi-domain systems for power system analysis have been created (Nicolet, Sapin, Simond, Prenat, \& Avellan, 2001; Sapin, 1995), however they do not support power grid modeling for stability-analysis or the capability to simulate large grids.

The authors of (Vanfretti et al., 2013) and (Gómez, Vanfretti, \& Olsen, 2015) have shown that Modelica language is able to cope with the exposed ambiguous model sharing issue while facilitating the access and/or modification of models at the "equation-level". Some additional advantages of Modelica are the open distribution of several libraries meant to represent physical systems, and the fact that models are independent from IDEs and solvers (Gómez et al., 2015). In addition, Modelica tools are now supporting the required numerical techniques to simulate large power grids (Braun, Casella, \& Bachmann, 2017; Casella, Leva, \& Bartolini, 2017; Dassault Systemes, 2018).

In addition, because turbine manufacturers already make extensive use of Modelica for thermo-mechanical and control modeling of gas turbines (Johansson, 2016), it becomes attractive to adopt a multi-domain modeling approach using Modelica in order to enhance dynamic characteristics of gas turbines and the power system..

\subsection{Contributions}

The work reported in this paper was carried out within the ITEA3 OpenCPS (Open Cyber-Physical System Model-Driven Certified Development) project. The project aims to develop modeling and simulation toolchains that can be applied to cyber-physical and multi-domain systems (ITEA3, 2017).

In the second use case of the work package D5.3B, the benchmark case corresponds to multi-domain models of improved gas turbines coupled to the power grid to meet European standardization requirements for grid connection.

This paper presents the development of a multidomain gas turbine and power grid equation-based model, required to test the functionalities of the OpenCPS toolchains. In more detail, first a Modelica multi-domain model comprising the physical model of the gas turbine, the governor and a Single Machine Infinite Bus (SMIB) power network was generated. Then, an analysis of the multi-domain system that includes a comparison with the GGOV1-based equivalent system was performed.
The modeling approach shown through the example of the integration of a multi-domain model of a gas turbine with the electric grid can be adopted in future scenarios of multi-domain power plant-to-network integration where more complex models of geothermal, combined-cycle or wind power plants, among other resources are being used. Power systems analysts will benefit from the availability of more accurate models as high penetration of intermittent renewable resources continues to challenge traditional power system operations, making their study difficult with traditional power system tools.

The paper is organized as follows: Section 1 provides a motivation to the problem, along with the previous work and contributions from this work. Section 2 starts with a description of the Modelica libraries used to develop the models. Subsequently, it continues with the presentation of the turbo-machinery and power system domain models, as well as the multi-domain model that is obtained by combining the models of the two previous domains. Section 3 explains the studies and simulations that have been carried out on the power system-only model and the multi-domain model for comparison purposes. Finally, Sections 4 and 5 present the results obtained and provide the conclusions, respectively.

\section{Equation-Based Models}

\subsection{Modeling Background}

\subsubsection{Package Structure}

The equation based models were built or/and modified inside of a package structure in the Dymola F2016 Modelica IDE. The adopted package structure was conceived to classify the models in terms of the domain they belong to. The first two packages, namely TurboMachineryDomain and PowerSystemDomain, contain the physical gas turbine models and the electric power system models, respectively. A third package, called MultiDomain, comprises the results of merging components from the two former packages to obtain the multi-domain equation based models. In PowerSystemDomain only components from the OpenIPSL library are included. In addition to the SMIB network models, new stochastic variable load model and the gas turbine controls based on the GGOV1 model are provided. On the other hand, the TurboMachineryDomain package was developed to comprise only elements from libraries specialized in gas turbines and other thermal power generation technologies such as ThermoPower (Casella \& Leva, 2003), ThermoSysPro (El-Hefni, Bouskela, \& Lebreton, 2011), ThermoFluid (Idebrant et al., 2003) or the ThermalPower library (Hübel et al., 2014). In this work only ThermoPower has been used. 


\subsubsection{OpenIPSL Library}

OpenIPSL is an open-source Modelica library that can be used to create power system networks and then perform dynamic time-domain simulation. The Modelica language provides to this library the flexibility that is not common to find in other power system modeling and simulation tools (Baudette et al., 2018).

\subsubsection{ThermoPower Library}

ThermoPower is an open-source Modelica library developed at Politecnico di Milano. It provides components that can be used to model thermal power plants (Casella \& Leva, 2003) (Casella, 2009).

The library has a package called Gas which contains the models of the gas turbine compressor, expansion turbine and combustion chamber. Their modeling description is based on DAE that are widely accepted in the turbine technology domain (Razak, 2007; Walsh \& Fletcher, 2004).

More information about the library can be found in the official website (see URL: https://casella.github.io/ThermoPower).

\subsection{Turbo-Machinery Domain Modelling}

The TurboMachineryDomain package contains models which employ ThermoPower components. Its contents are organized in 3 sub-packages, namely GTArrangements, GTModels and Tests.

\subsubsection{The GTArrangements package}

As the name implies, the first package aimed to include the elementary gas turbine topologies. The SingleShaftGT model represents a single shaft gas turbine and it is based on the Plant model of the Brayton Cycle examples of ThermoPower. The Brayton Cycle is the thermodynamic cycle that describes the operation of a gas turbine. It is composed of at least four processes, three of which are associated with the components of a gas turbine, namely: compressor, combustion chamber and expansion turbine. The model only focuses on the internal components of the gas turbine. The parameters of the compressor, combustion chamber and turbine are propagated and therefore, the SingleShaftGT can be used as a generic block in the representation of gas power plants.

\subsubsection{The GTModels package}

The second package has the models that result from combining the basic parametrized gas turbine arrangement with given boundary conditions, sensors and actuators. The only example included to date is the complete ThermoPower Single Shaft Gas Turbine ThPowerSSGT model, which can be seen in Figure 1.

Due to unavailability of data, the design parameters and component characteristics of the ThPowerSSGT gas turbine model were not modified with respect to the ones of the original ThermoPower example. However, a still simple but complete model of the fuel inlet valve that takes valve position as input instead of fuel mass flow reference was added. This change was needed to harmonize the physical model of the turbine with the simplified power system GGOV1-based turbine model.

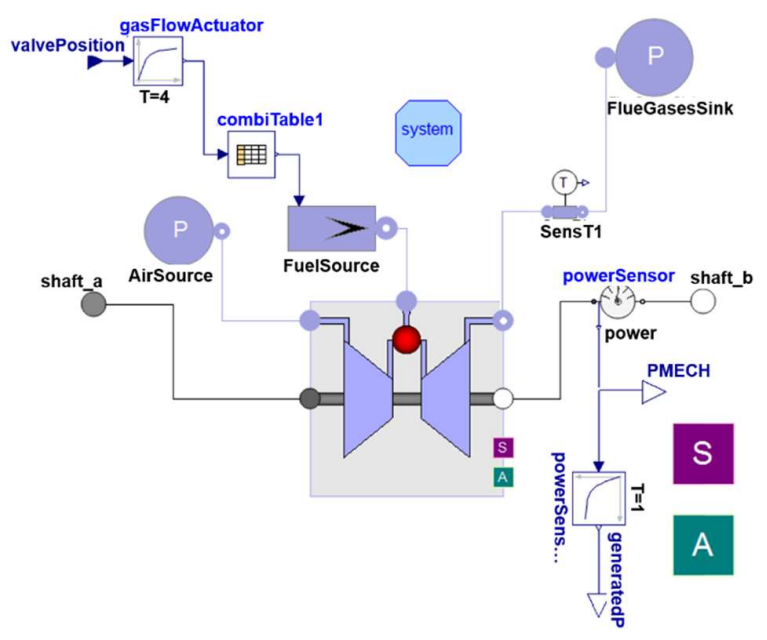

Figure 1. The Single Shaft Gas Turbine model built using ThermoPower components.

In order to build a valve model compatible to the simplified representations used in power system analysis, in particular the GGOV1 model, the fuel mass flow rate $\dot{m}_{\text {fuel }}$ values required to obtain mechanical power from 0 to the maximum value of $10 \mathrm{MW}$ were obtained through simulations and are shown in Table 1. In power system simulations, $P_{\text {mech }}$, is used as the output of the turbine. Hence, to relate the output mechanical power $P_{\text {mech }}$ (in per unit) with the fuel inlet valve position $\theta_{\text {fuel valve }}$ (also in per unit), the following expression was used:

$$
\theta_{\text {fuel valve }}=P_{\text {mech }} / K_{\text {turb }}+W_{f n l}
$$

where $K_{\text {turb }}$ is the gas turbine gain and $W_{f n l}$ is the fuel mass flow rate at no load conditions (in per unit). Evaluating the parameters for a range of $0-10 \mathrm{MW}$ gives a look-up table whose values are shown in Table 1.

Table 1. Fuel inlet valve model design data with $\boldsymbol{K}_{\text {turb }}=$ 1.5 and $\boldsymbol{W}_{\text {fnl }}=0.15$.

\begin{tabular}{|c|c|c|c|}
\hline $\begin{array}{c}P_{\text {mech }} \\
(\mathrm{MW})\end{array}$ & $\begin{array}{c}P_{\text {mech }} \\
(\mathrm{pu})\end{array}$ & $\theta_{\text {fuel valve }}(\mathrm{pu})$ & $\begin{array}{c}\dot{m}_{\text {fuel }} \\
(\mathrm{kg} / \mathrm{s})\end{array}$ \\
\hline 0 & 0 & 0.150 & 1.845 \\
\hline 1 & 0.1 & 0.217 & 1.919 \\
\hline 2 & 0.2 & 0.283 & 1.989 \\
\hline 3 & 0.3 & 0.350 & 2.059 \\
\hline 4 & 0.4 & 0.417 & 2.129 \\
\hline 5 & 0.5 & 0.483 & 2.199 \\
\hline 6 & 0.6 & 0.550 & 2.270 \\
\hline 7 & 0.7 & 0.617 & 2.341 \\
\hline 8 & 0.8 & 0.683 & 2.413 \\
\hline 9 & 0.9 & 0.750 & 2.485 \\
\hline 10 & 1.0 & 0.817 & 2.558 \\
\hline
\end{tabular}


Finally, the curve $\dot{m}_{f u e l}=f\left(\theta_{\text {fuel valve }}\right)$ from Table 1 was specified in the model as the look-up table that is shown in Figure 1 (see combiTable1D).

\subsection{Power System Domain Modelling}

This section provides an overview of the grid, load and control models which are based on OpenIPSL library components.

\subsubsection{Generation Groups}

The benchmark multi-domain model of use case 2 of the OpenCPS project work package (i.e. improved gas turbines coupled to the power grid) required different modeling and simulation scenarios for the SMIB network model. Two examples of these scenarios are a SMIB model without controls and a SMIB model with only excitation system. They were made available inside the sub-package Generation_Groups of the PowerSystemDomain package. This sub-package also includes a model of the "infinite bus" modeling construct which is typically used in power systems to represent a strong external system.

\subsubsection{Controls}

The GGOV1 is one of the so-called turbine-governor models that analysts use in power system dynamic studies. This is a generic model with blocks to represent thermal turbines that are controlled by a PID governor (proportional, integral, derivative). It also includes blocks that represent the dynamics of the fuel system, acceleration limiter, load limiter by exhaust temperature control, valve position and supervisory load controller.

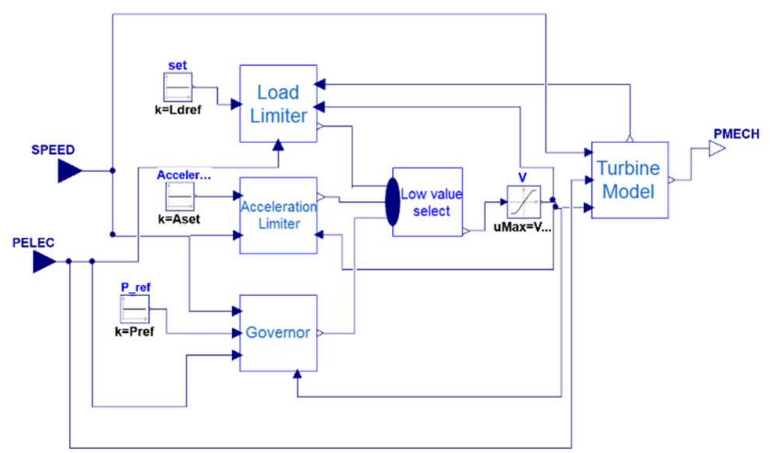

Figure 2. Modified GGOV1 Turbine Governor model.

The GGOV1 model implementation of the OpenIPSL library was refactored (i.e. modularized functions into internal blocks) so to fit the needs of the studies of this work.

As shown in Figure 2, refactoring was applied on the GGOV1 model to explicitly show its internal functionalities using internal blocks. This means that a separate model was created for each of the three controls logics that are inside of the GGOV1 model, namely the load limiter, the acceleration limiter and the main governor. Another model was developed to represent only the turbine, thus obtaining a convenient way to re- use the models when a certain study requires to modify an internal block (e.g. only the turbine or the governor) instead of the entire model.

\subsubsection{Network Models}

A SMIB network model was developed for each of the generation groups described in Section 2.3.1. Figure 3 shows the SMIB network case where the generation group has no controls.

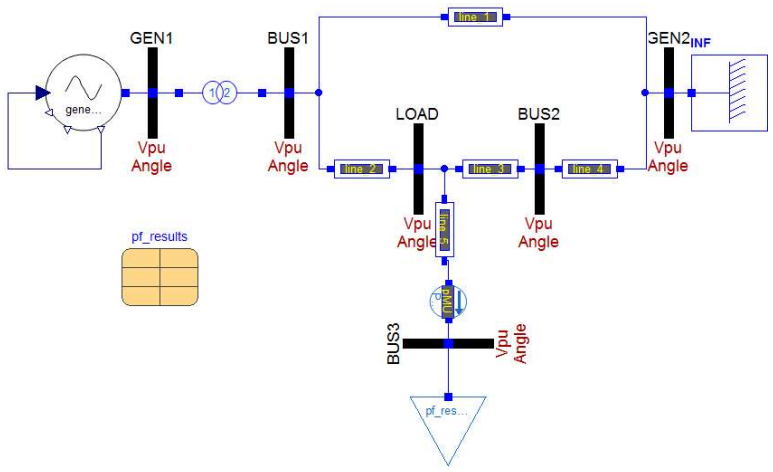

Figure 3. SMIB network model with no turbine governor model.

The initial voltage magnitude, voltage angle, active power and reactive power values of the generators, the load and the buses are specified by means of the record pf results, which were obtained using and identical representation of the network using $\mathrm{PSS} / \mathrm{E}$, a domain specific tool (Siemens AG, 2018).

\subsubsection{Variable Load Model}

As it can be noticed from Figure 3, the SMIB network model also includes a variable load component. It can behave deterministically or stochastically, where the latter requires a stochastic signal as an input.

This model has now been included in the OpenIPSL library as OpenIPSL.Electrical.PSSE.Load_ExtInput. It is similar to the ./Load variation model, with the main difference being that it has a real input for modulation Error! Reference source not found.. Therefore, the new model has a component that allows for active power modulation in addition to the component that represents the physical load variability. The second component is

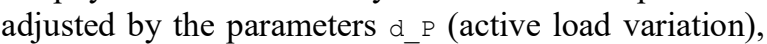
$d t$ and $t 1$ (start time and time duration of load variation), while the former relies on the noise injection source that is connected to this model (through input $\mathbf{u}$ ). Noise injections have also been included in OpenIPSL, and are available under OpenIPSL.Electrical.Loads.PSSE.NoiseInjections The simulation results where the load models exhibit a stochastic behavior driven at $\mathbf{u}$ are out of the scope of this paper, (see (Aguilera, Vanfretti, \& Gómez, 2018)). 


\subsection{Multi-Domain Model}

A SMIB network model and a governor block model from the PowerSystemDomain package was combined with the physical model of a gas turbine from the TurboMachineryDomain package. The result of this procedure gives the so-called multi-domain model that can be appraised in Figure 4.

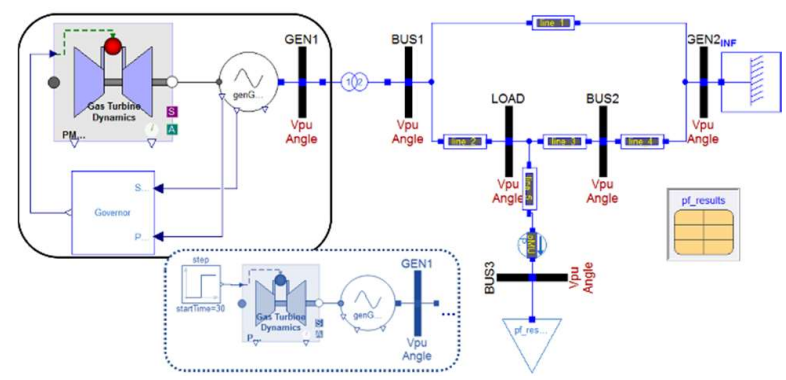

Figure 4. Multi-domain SMIB model.

New generation group sets have been created to allow the connection between the generator and the detailed gas turbine model. Even though these groups still rely on the previously defined groups of the PowerSystemDomain package (see Section 2.3.1), they also include an interface block. The function of this new block is to relate the rotational mechanics (flange internal variables) of the gas turbine model with the generator mechanical power and speed.

\section{Simulation Studies}

In this section, the simulations and studies that were applied on the equation-based models will be described. They include the identification of a GGOV1 turbine model, a frequency-domain analysis of the gas turbine models and simulations of the models when they are subject to a load change. An emphasis has been placed on the comparative analysis between the multi-domain model and the model that uses GGOV1 model that is a power system domain aggregate turbine-governor representation.

\subsection{Study and Simulation Cases}

This section begins with a description of the identification process of the GGOV1-based turbine model that fits the response of the ThermoPower detailed model. The results are necessary to carry out comparative studies on the resulting SMIB network models, namely a frequency domain analysis and the response to a load change event.

\subsubsection{GGOV1-based Turbine Model Identification}

The first step in the analysis to be done on the SMIB network models is the identification of the GGOV1 turbine model that is equivalent, in terms of its openloop time response, to the ThermoPower model. An open-loop test has been applied to the multi-domain SMIB model for that purpose.

The governor has been removed from the multidomain SMIB model to apply a step change on the fuel mass flow rate in the gas turbine model. This can be observed in the box with dotted line of Figure 4, that replaces the box with solid line. Table 1 was employed to find the fuel mass flow rate values that give an output mechanical power change from 5 to $8 \mathrm{MW}$. Subsequently, a simulation was carried out in Dymola with a duration of 100 seconds, where the step change occurred after 30 seconds. The results were saved to proceed with the identification of the GGOV1-based turbine model.

The simulation output data has been imported in MATLAB as a .mat file. Then the system identification ident tool has been used to fit a GGOV1-turbine model that suits the reference model. The values of turbine gain $K_{t u r b}$ and the no load fuel flow $W_{f n l}$ were set to 1.5 and 0.15 , respectively, as explained in Section 2.2.2. Additionally, the damping factor $D_{m}$ was set to the typical value of 0 . The decision to not consider this parameter is also based on the argument of (Pourbeik, 2013) that states: "A speed damping factor can be modeled to influence the temperature limit as a rather gross approximation of the speed dependence of the turbine rating. This is, however, not very accurate". Thus, it has only been required to obtain the values of the parameters of the lead-lag transfer function $T_{b}$ and $T_{c}$, together with the delay transport time $T_{\text {eng }}$.

From now on, the SMIB model that contains the physical model of the turbine will be referred to as multi-domain model. On the other hand, the SMIB network model using the GGOV1-based turbine model will be referred to as power system-only model.

\subsubsection{Gas Turbine Models Frequency Domain Analysis}

A first inspection of the differences between the expected response of the multi-domain model and the power system-only model can be carried out through pole/zero analysis. This study requires the linearization of the physical turbine model around a given operating point, which can be performed automatically from Dymola using the Modelica Linear Systems 2 library (Baur, Otter, \& Thiele, 2009).

To understand the impact of the eigenvalues on the response of each model, the contribution of the poles in the states obtained after linearization has been identified. The results are discussed in Section 3.2.2.

\subsubsection{Load Change Event Simulations}

The next step is to verify the time-domain response of the models under a load change.

A simulation of 100 seconds was performed on both the multi-domain and power-system model (using the identified parameters as described in Section 3.1.1), 
with the same governor model. The active power of the load was increased by $0.2 \mathrm{pu}$ after 30 seconds of simulation, and was set back again to the original value after 20 seconds, in order to evaluate the turbine response to a sudden load change.

Successive simulations were carried out with the objective of evaluating the performance of the models at different operating points. Specifically, the load active power as well as the dispatched power from the generator were increased from 5 to $9.8 \mathrm{MW}$, in steps of $0.1 \mathrm{MW}$.

The load active power and dispatched generator power parameter sweep required the computation of 49 power flow solutions for the initialization of the network models. The solution sets were supplied in the form of records to conform with the description of Section 2.3.3. Python scripts were used to automatically generate the Modelica records. The scripts consist of a modified version of the toolset used to get the Nordic 44-bus system simulation results published in (Vanfretti, Rabuzin, Baudette, \& Murad, 2016) and (Vanfretti et al., 2017).

In order to better quantify the time domain response differences of the two models, the settling times were computed. This was performed for the first simulation scenario, when the load model did not include the noise. Results are presented in Section 3.2.3 and discussed in Section 4.

\subsection{Results}

The results of the studies and simulations performed on the models are presented in this section.

\subsubsection{Model Identification}

A GGOV1-based turbine model with one pole and one zero with no time delay was identified. The resulting transfer function is:

$$
g_{4}(s)=K_{\text {turb }} \frac{1+T_{c} s}{1+T_{b} s}=1.5 \cdot \frac{1+0.115 s}{1+0.141 s}
$$

The same step change on the fuel mass flow rate was applied on both the reference multi-domain model and the power system-only model without the governor. Figure 5 shows the output mechanical power plots from the turbine components of the models.

\subsubsection{Eigenanalysis}

The gas turbine models can be described in state space form as:

$$
\begin{gathered}
\dot{x}=A x+B u \\
y=C x+D u
\end{gathered}
$$

In the case of the GGOV1-based turbine system of the Power System-only model, the system vectors and matrices are defined as:

$$
\text { u:valve } \text { position } \quad y: P_{\text {mech }}
$$

$$
x=\left(\begin{array}{c}
g_{4}(s) \cdot x \\
\text { gasFlowActuator } . y
\end{array}\right)
$$

$$
\begin{array}{cc}
\boldsymbol{A}=\left(\begin{array}{cc}
-7.092 & 11.123 \\
0 & -0.25
\end{array}\right) & \boldsymbol{B}=\left(\begin{array}{c}
0 \\
0.25
\end{array}\right)^{-1} \\
\boldsymbol{C}=\left(\begin{array}{c}
1.835 \times 10^{6} \\
12.807 \times 10^{6}
\end{array}\right) & \boldsymbol{D}=0
\end{array}
$$

It can be easily found from the system state space equation that there are only real eigenvalues. They are shown on Table 2 together with their contribution on the identified systems states.

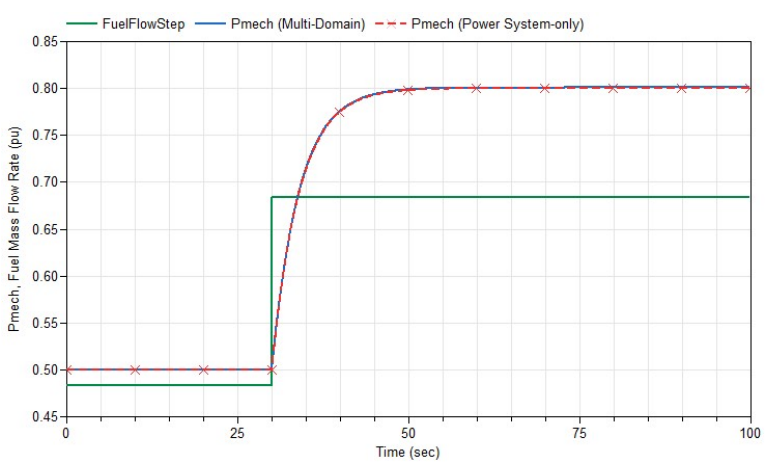

Figure 5. Open-Loop test to verify the response of the identified model, w.r.t. the multi-domain model.

Table 2. Real eigenvalues of gas turbine in the power system-only model.

\begin{tabular}{|c|c|c|c|}
\hline \multirow{2}{*}{ Eigenvalue } & \multirow{2}{*}{$T(s)$} & \multicolumn{2}{|c|}{ Contribution to states } \\
\cline { 3 - 4 } & & State & $\begin{array}{c}\text { Contribution } \\
(\%)\end{array}$ \\
\hline$p_{1}=-7.092$ & 0.141 & $g_{4}(s) \cdot x$ & 100 \\
\hline$p_{2}=-0.25$ & \multirow{2}{*}{4.0} & $g_{4}(s) \cdot x$ & 61.9 \\
\cline { 3 - 4 } & & gasFlowActuator. $y$ & 38.1 \\
\hline
\end{tabular}

The system has only a zero $z_{1}=-8.685$ with $T(s)=$ 0.115 .

Linearization was performed on the detailed gas turbine system of the multi-domain model at $\mathrm{t}=0$. The state vector is as follows:

$$
\begin{gathered}
x=[\text { CC.fluegas. } p, \quad \text { CC.fluegas. } T, \\
\text { CC.fluegas. } X_{1}, \text { CC.fluegas. } X_{2}, \\
\text { CC.fluegas. } X_{3}, \text { CC.fluegas. } X_{4}, \\
\text { CC.fluegas. } X_{5}, \quad \text { CC. } T_{m}, \\
\text { gasFlowActuator. } y, \text { speedSource. } \phi \\
\text { gasFlowActuator. } y, \text { speedSource. } \phi]^{T}
\end{gathered}
$$

As can be seen in equation 6 , most system states are associated with the combustion chamber of the turbine (CC). These states are the chamber wall temperature (Tm) and the pressure $(p)$, temperature $(T)$ and molar composition $\left(X_{1}-X_{5}\right)$ of the gases at the chamber outlet (i.e. flue gases).

System vectors $\mathrm{B}$ and $\mathrm{C}$ are now defined as follows:

$$
\boldsymbol{B}=\left[\begin{array}{llllllllll}
0 & 0 & 0 & 0 & 0 & 0 & 0 & 0 & 0.25 & 0
\end{array}\right]^{T}
$$




$$
\begin{array}{rlr}
\boldsymbol{C}=[78.860, & 20707.3, & 3.939 \times 10^{7}, \\
2.918 \times 10^{7}, & 7.291 \times 10^{7}, \\
3.163 \times 10^{7}, & 4.456 \times 10^{7}, \\
& 0,0,0]
\end{array}
$$

Matrix A is given by:
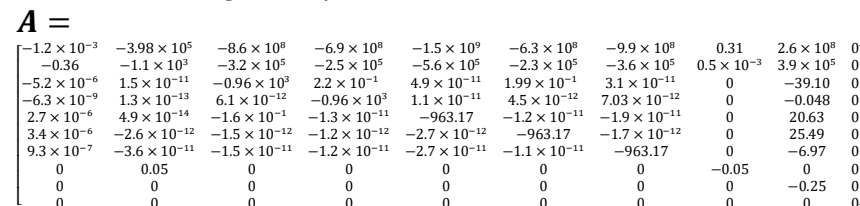

The system has also only real eigenvalues as it is

\begin{tabular}{|c|c|c|c|}
\hline \multirow{2}{*}{ Eigenvalue } & \multirow{2}{*}{$T(s)$} & \multicolumn{2}{|c|}{$\begin{array}{c}\text { Relevant contribution to } \\
\text { states }\end{array}$} \\
\hline & & State & $\begin{array}{c}\text { Contribution } \\
\text { (\%) }\end{array}$ \\
\hline$p_{1}=-1.56 \times 10^{3}$ & $6 \times 10^{-4}$ & CC.fluegas.p & 99.9 \\
\hline$p_{2}=-9.63 \times 10^{2}$ & 0.001 & CC.fluegas.T & 97.2 \\
\hline \multirow{2}{*}{$p_{3}=-9.63 \times 10^{2}$} & \multirow{2}{*}{0.001} & CC.fluegas.T & 75 \\
\hline & & CC.fluegas. $X_{\text {具 }}$ & 12.2 \\
\hline$p_{4}=-9.63 \times 10^{2}$ & 0.001 & CC.fluegas.T & 94.8 \\
\hline \multirow{2}{*}{$p_{5}=-9.63 \times 10^{2}$} & \multirow{2}{*}{0.001} & CC.fluegas.T & 86.4 \\
\hline & & CC.fluegas. $X$ & 5.5 \\
\hline$p_{6}=-9.63 \times 10^{2}$ & 0.001 & CC.fluegas.T & 99.4 \\
\hline$p_{7}=-7.98 \times 10^{2}$ & $\begin{array}{l}1.3 \times \\
10^{-3} \\
\end{array}$ & CC.fluegas.p & 99.9 \\
\hline$p_{8}=-0.25$ & 4.000 & CC.fluegas.p & 99.6 \\
\hline$p_{9}=-0.05$ & 20.00 & $C C . T_{m}$ & 100 \\
\hline$p_{10}=0$ & -- & speedSource. & 100 \\
\hline
\end{tabular}
shown in Table 3.

Table 3. Real eigenvalues of gas turbine in the multidomain model.

Finally, the gas turbine of the multi-domain model has the zeroes shown in Table 4. The poles and zeros plots of the gas turbine systems from both models can be observed in Figures 6 and 7.

Table 4. Zeros of the gas turbine in the multi-domain model.

\begin{tabular}{|c|c|c|}
\hline Zero & Amount & $T(\mathrm{~s})$ \\
\hline$z_{1}=-9.644 \times 10^{2}$ & 1 & 0.001 \\
\hline$z_{i}=-9.632 \times 10^{2}$ & 4 & 0.001 \\
\hline$z_{6}=-6.441 \times 10^{2}$ & 1 & $1.6 \times 10^{-3}$ \\
\hline$z_{7}=-0.050$ & 1 & 20.000 \\
\hline$z_{8}=-6.651 \times 10^{-14}$ & 1 & $1.503 \times 10^{13}$ \\
\hline
\end{tabular}

Table 5. Branch data of the SMIB network model $\left(\boldsymbol{V}_{\boldsymbol{b}}=\right.$ $13.8 \mathrm{kV})$.

\begin{tabular}{|c|c|c|c|}
\hline From bus & To bus & $R(p u)$ & $X(p u)$ \\
\hline GEN1 & BUS1 & 0 & 0.150 \\
\hline BUS1 & GEN2 & $1 \times 10^{-5}$ & 0.200 \\
\hline BUS1 & LOAD & $3 \times 10^{-5}$ & 0.060 \\
\hline LOAD & BUS2 & $3,5 \times 10^{-4}$ & 0.070 \\
\hline BUS2 & GEN2 & $3,5 \times 10^{-4}$ & 0.070 \\
\hline LOAD & BUS3 & 0 & $1 \times 10^{-5}$ \\
\hline
\end{tabular}

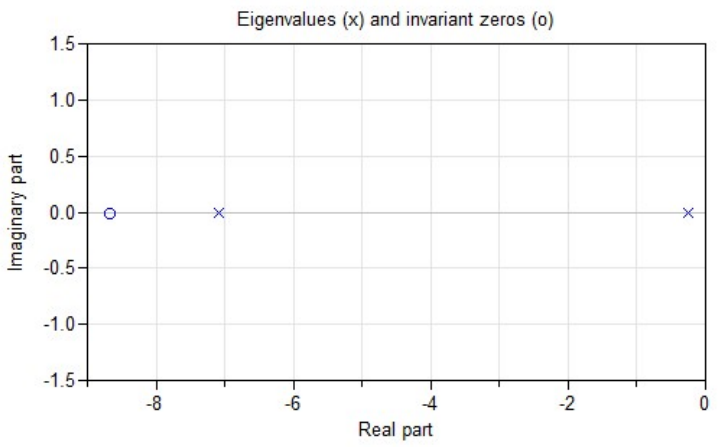

Figure 6. Poles and zeros of the gas turbine in the power system-only model.

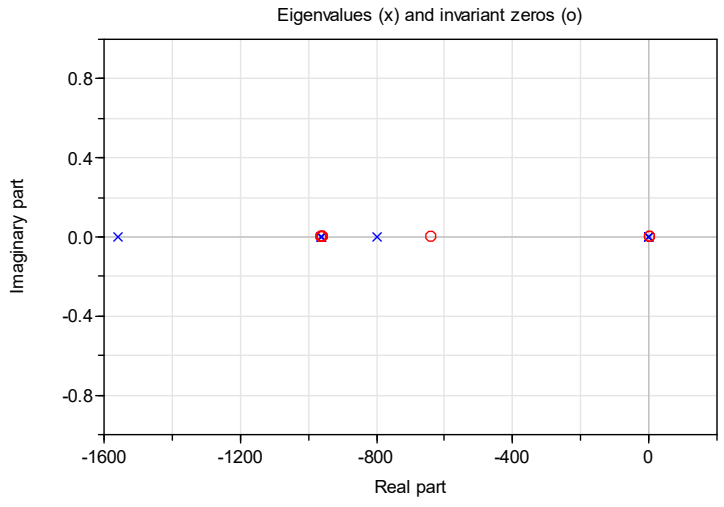

Figure 7. Poles and zeros of the gas turbine in the multidomain model.

\subsubsection{Time Response to Load Change}

The governor was added to the multi-domain and power system-only models to evaluate their time response to a load change. The applied test was presented in Section 3.1.3 and the model parameters can be found in Tables 5-7. This section shows the results of the time response simulations. The simulations were performed with the variable step DASSL solver and a tolerance of $1 \times 10^{-4}$.

Table 6. Parameters of generated at BUS1 $\left(\boldsymbol{V}_{\boldsymbol{b}}=13.8 \mathrm{kV}\right)$.

\begin{tabular}{|c|c|c|c|}
\hline Parameter & Gen-1 & Parameter & Gen-1 \\
\hline Capacity $M_{b}$ & $10 \mathrm{MVA}$ & $X_{q}$ & 1.35 \\
\hline$T_{d 0}^{\prime}$ & 5.00 & $X_{d}^{\prime}$ & 0.30 \\
\hline$T_{d 0}^{\prime \prime}$ & 0.05 & $X_{q}^{\prime}$ & 0.60 \\
\hline$T_{q 0}^{\prime}$ & 0.70 & $X_{d}^{\prime \prime}=X_{q}^{\prime \prime}$ & 0.20 \\
\hline$T_{q 0}^{\prime \prime}$ & 0.10 & $X_{l}$ & 0.12 \\
\hline Inertia H & 4.00 & $S_{1.0}$ & 0.10 \\
\hline Damping D & 0 & $S_{1.2}$ & 0.50 \\
\hline$X_{d}$ & 1.41 & $R_{a}$ & 0 \\
\hline
\end{tabular}

Figure 8 shows a plot of the mechanical power delivered by the gas turbine components. Special attention was also given to the response of the system frequency and the electrical power of the generator. The corresponding plots can be seen in Figures 9 and 10, respectively. 
Then the simulation was repeated several times to measure the settling times, at different operating points of the generator. Figure 11 first shows a plot of the calculated settling times in both multi-domain and power system-only models as a function of the load/generator active power. The plot at the bottom of the figure shows the difference between the settling times obtained for the power system-only model and the settling times of the multi-domain model.

Table 7. GGOV1 governor-only parameters.

\begin{tabular}{|c|c|c|c|}
\hline Parameter & Value & Parameter & Value \\
\hline$R(\mathrm{pu})$ & 0.04 & $K_{\text {turb }}(\mathrm{pu})$ & 1.50 \\
\hline$T_{\text {pelec }}(\mathrm{sec})$ & 1.00 & $D_{m}(\mathrm{pu})$ & 0.00 \\
\hline $\max _{\text {err }}(\mathrm{pu})$ & 0.05 & $K_{\text {imw }}, d b(\mathrm{pu})$ & 0.00 \\
\hline $\min _{\text {err }}(\mathrm{pu})$ & -0.05 & $V_{\max }(\mathrm{pu})$ & 1.00 \\
\hline$K_{\text {pgov }}(\mathrm{pu})$ & 10.00 & $V_{\min }(\mathrm{pu})$ & 0.10 \\
\hline$K_{\text {igov }}(\mathrm{pu})$ & 5.00 & $W_{\text {fnl }}(\mathrm{pu})$ & 0.15 \\
\hline$K_{\text {dgov }}(\mathrm{pu})$ & 0.00 & FLAG & 0 \\
\hline$T_{\text {dgov }}(\mathrm{sec})$ & 1.00 & \multicolumn{3}{|c}{} \\
\cline { 1 - 3 } & \multicolumn{1}{|l}{}
\end{tabular}

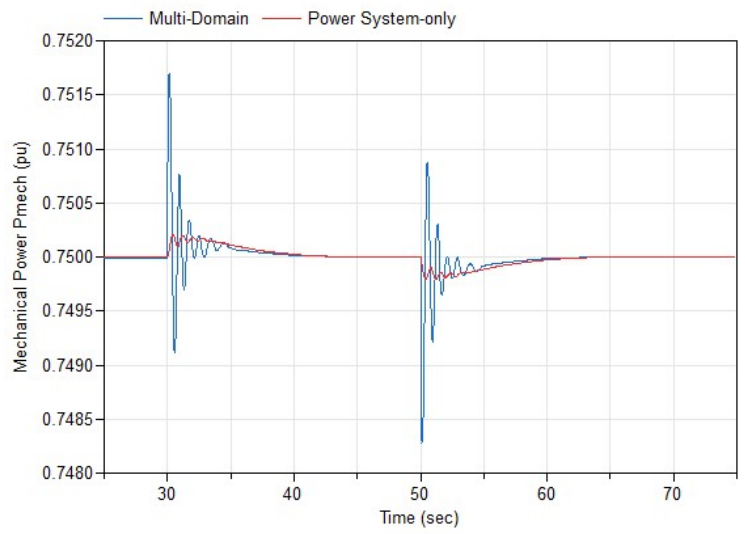

Figure 8. Mechanical power response comparison.

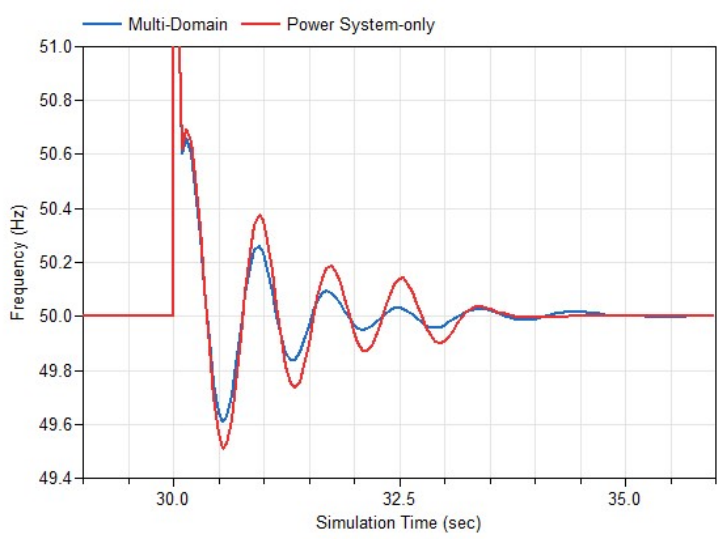

Figure 9. Frequency response comparison.

\section{Discussion}

The first evidence of the differences between the power system-only model and the multi-domain models can be found in the frequency analysis results from Section 3.2. The higher number of poles and states identified in the linearized multi-domain model shows that the GGOV1 model used in the power system-only model will lead to loss of information about the physical dynamics of the turbine.

The information reported in Table 3 is particularly useful when it comes to giving a better physical explanation to the behavior of the model. First, the list of states allows to appreciate the relevance of the heating process in the gas turbine dynamics. Seven out of nine states were related to thermodynamic properties in the boundaries of the combustion chamber (denoted as $\mathrm{CC}$ in the state vector). However, six poles are cancelled with zeros, and thus only three poles deserve special attention.

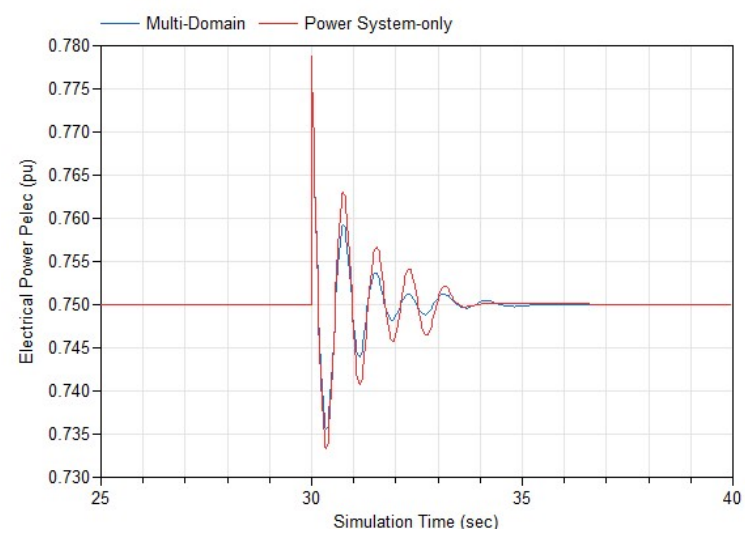

Figure 10. Electrical power response comparison.
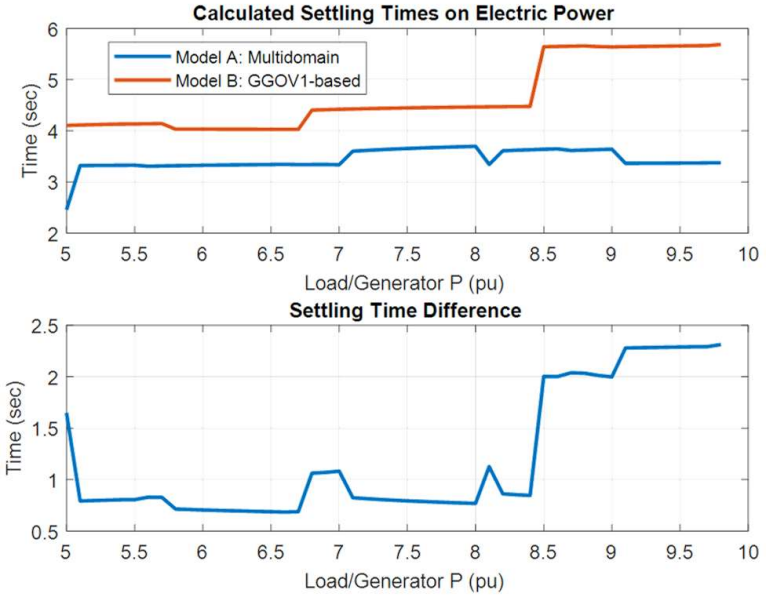

Figure 11. Calculated settling times in the electrical power response.

One of those poles is related to the fuel system actuator transfer function, which is also present in the simpler GGOV1-based model. As it can be seen in Table 3 , the other two poles have a high contribution in the pressure of the flue gases at the exhaust of the combustion chamber. From theory, thermodynamic state of a gas is determined by two properties such as pressure and temperature, in addition to the molecular composition. The pressure of the flue gases (i.e. the 
relevant state in the detailed gas turbine model) is directly linked to the Brayton Cycle and to the turbine's operation characteristics.

Figures 6 and 7 together with Tables 2 and 3 lead to another significant finding. In general, the explicit model from ThermoPower provides a higher bandwidth resolution in behavior modeling that is not possible with the GGOV1-based model. Therefore, this shows how a multi-domain model will be more suitable for transient stability studies (e.g. fault analysis, control design, etc.)

The effects on the time response of the models can be first examined in Figures 8 to 10. The load change event influences the system frequency, which is measured closed to the load bus, and is shown in Figure 9. Even if it is for a short time (around $2 \mathrm{sec}$ ), the frequency experiences a maximum deviation of up to $1 \mathrm{~Hz}$. Such frequency excursions are unacceptable in practice as protective over/under frequency protection systems can be triggered. Observe that the power system-only model results give an over-estimation of the expected frequency, and thus, any control/protection system design using such model may give unexpected results in practice. In Figure 9, the frequency of the power systemonly model goes beyond $49.6 \mathrm{~Hz}$ which is typically the limit for under-frequency protections, while the multidomain model is below it, making the latter more suitable for model-based design.

The GGOV1 turbine model is not dependent on the shaft speed and therefore, the changes on the mechanical power of Figure 8 are due to the governor's response. However, this is not in the case of the multi-domain turbine model. That explains why the model produces an additional oscillatory behavior on the mechanical power that cannot be observed in the GGOV1-based turbine model response. Also, note that the output mechanical power is grossly under-estimated by the power system-only model w.r.t the multi-domain model.

The electrical power can be used to examine the impact of the gas turbine model response on the generator's electric power output (see Figure 10). Nevertheless, it is important to keep in mind that the electrical power is also directly influenced by the speed. The settling times of this variable were calculated for different values of the load/generator power and then plotted in Figure 11. The results show a higher amplitude in the frequency response when the GGOV1based turbine model is employed. Although the settling times difference between the two models' response keep fairly constant, an increase is obtained for active power values greater or equal than $0.85 \mathrm{pu}$. It has been found that the cause of this performance is the saturation of the fuel actuator limiter in the GGOV1-based turbine model.

\section{Conclusions}

The following conclusions and recommendations can be drawn from this work:
- A multi-domain model has been derived to allow simulations of detailed representations of gas turbines and the electric power grid. Although the models are simple (due to the lack of available modeling information) the methodology provides a framework for future studies with multi-domain models in power systems.

- Differences in the simple turbine model (GGOV1) and the multi-domain explicit turbine model have been shown. A relevant source of that difference is the representation of the speed influence on the gas turbine dynamics. The study was, however, limited by the lack of measurements that could have served as a reference for the model's tuning and validation. It would also be of value to analyze the differences between the models in other power network variables and not only in the generator response.

This work gives a proof-of-concept on the use of Modelica for joint modeling of complex energy sources without the loss of information that traditional power system approaches incur in. The multi-domain approach is thus valuable for power system analysts, especially those dealing with controller design and dynamic performance analysis.

\section{Acknowledgement}

The authors would like to thank Francesco Casella for his assistance with questions regarding ThermoPower. In the same way, they would like to acknowledge the contributions of Tin Rabuzin in initial stage of this work.

\section{References}

Aguilera, M., Vanfretti, L., \& Gómez, F. (2018). Experiences in power system multi-domain modeling and simulation with modelica \& FMI: The case of gas power turbines and power systems. In 2018 Workshop on Modeling and Simulation of Cyber-Physical Energy Systems (MSCPES) (pp. 1$6)$. https://doi.org/10.1109/MSCPES.2018.8405397

Baudette, M., Castro, M., Rabuzin, T., Lavenius, J., Bogodorova, T., \& Vanfretti, L. (2018). OpenInstance Power System Library Update 1.5 to "iTesla Power Systems Library (iPSL): A Modelica library for phasor time-domain simulations. SoftwareX. https://doi.org/10.1016/j.softx.2018.01.002

Baur, M., Otter, M., \& Thiele, B. (2009). Modelica Libraries for Linear Control Systems LinearSystems library. Proceedings of the 7th International Modelica Conference, 20-22.

Braun, W., Casella, F., \& Bachmann, B. (2017). Solving large-scale Modelica models: new approaches and experimental results using OpenModelica. In Proceedings of the 12th International Modelica Conference, Prague, Czech Republic, May 15-17, 2017 (pp. 557-563). 
Carnegie Mellon University. (2013). Managing Variable Energy Resources to Increase Renewable Electricity's Contribution to the Grid.

Casella, F. (2009). Object-oriented modelling of power plants: a structured approach. IFAC Proceedings Volumes, 42(9), 249-254.

Casella, F., \& Leva, A. (2003). Modelica open library for power plant simulation: design and experimental validation. In Proceeding of the 2003 Modelica conference, Linkoping, Sweden.

Casella, F., Leva, A., \& Bartolini, A. (2017). Simulation of Large Grids in OpenModelica: reflections and perspectives, 227-233.

CEN-CENELEC-ETSI Smart Grid Coordination Group. (2012). Smart Grid Reference Architecture, (November), 1-46. Retrieved from $\mathrm{ftp}: / / \mathrm{ftp}$.cencenelec.eu/EN/EuropeanStandardization /HotTopics/SmartGrids/Reference_Architecture_fin al.pdf

Dassault Systemes. (2018). Dymola Sparse Solvers for Large-Scale Simulations.

De Mello, F. P., \& Ahner, D. J. (1994). Dynamic models for combined cycle plants in power system studies. IEEE Transactions on Power Systems, 9(3).

El-Hefni, B., Bouskela, D., \& Lebreton, G. (2011). Dynamic Modelling of a Combined Cycle Power Plant with ThermoSysPro. Proceedings of the 9th Modelica Conference, 365-375.

Gómez, F. J., Vanfretti, L., \& Olsen, S. H. (2015). Binding cim and modelica for consistent power system dynamic model exchange and simulation. In Power \& Energy Society General Meeting, 2015 IEEE (pp. $1-5)$.

Gomez, F., Vanfretti, L., \& Olsen, S. H. (2018). CIMCompliant Power System Dynamic Model-to-Model Transformation and Modelica Simulation. IEEE Transactions on Industrial Informatics, 3203(c), 11. https://doi.org/10.1109/TII.2017.2785439

Hannett, L. N., \& Khan, A. H. (1993). Combustion turbine dynamic model validation from tests. IEEE Transactions on Power Systems, 8(1), 152-158.

Hübel, M., Berndt, A., Meinke, S., Richter, M., Mutschler, P., Hassel, E., ... Funkquist, J. (2014). Modelling a lignite power plant in modelica to evaluate the effects of dynamic operation and offering grid services. In Proceedings of the 10 th International Modelica Conference; March 10-12; 2014; Lund; Sweden (pp. 1037-1046).

Idebrant, A., Näs, L., Ab, M. E., Industrial, A., Ab, T., Bachmann, B., ... Fritzson, P. (2003). Gas Turbine Applications using ThermoFluid. Proceedings of the 3rd International Modelica Conference.

IEA. (2016). Energy, Climate Change \& Environment 2016 Insights.

ITEA3. (2017). OpenCPS - Open Cyber-Physical System Model-Drive Certified Development. Retrieved June 19, 2017, from https://itea3.org/project/opencps.html

Johansson, T. (2016). Simulation of gas channel temperatures during transients for SGT-800.

Nicolet, C., Sapin, A., Simond, J. J., Prenat, J. E., \& Avellan, F. (2001). A new tool for the simulation of dynamic behaviour of hydroelectric power plants. In Proceedings of the 10th International Meeting of WG1, IAHR, Trondheim, Norway.

Pereira, L., Undrill, J., Kosterev, D., Davies, D., \& Patterson, S. (2003). A new thermal governor modeling approach in the WECC. IEEE Transactions on Power Systems, 18(2), 819-829.

Pourbeik, P. (2013). Dynamic models for turbinegovernors in power system studies. IEEE Task Force on Turbine-Governor Modeling.

Razak, A. M. Y. (2007). Industrial gas turbines: performance and operability. Elsevier.

Rowen, W. I. (1983). Simplified mathematical representations of heavy-duty gas turbines. Journal of Engineering for Power, 105(4), 865-869.

Rowen, W. I. (1992). Simplified mathematical representations of single shaft gas turbines in mechanical drive service. In ASME 1992 International Gas Turbine and Aeroengine Congress and Exposition (p. V005T15A001--V005T15A001).

Sapin, A. (1995). Logiciel modulaire pour la simulation et 1 " étude des systèmes d'entraînement et des réseaux électriques.

Siemens AG. (2018). PSS $® E$ - high-performance transmission planning and analysis software. Retrieved from https://www.siemens.com/1

Vanfretti, L., Li, W., Bogodorova, T., \& Panciatici, P. (2013). Unambiguous power system dynamic modeling and simulation using modelica tools. In Power and Energy Society General Meeting (PES), 2013 IEEE (pp. 1-5).

Vanfretti, L., Olsen, S. H., Arava, V. S. N., Laera, G., Bidadfar, A., Rabuzin, T., ... Gómez-López, F. J. (2017). An open data repository and a data processing software toolset of an equivalent Nordic grid model matched to historical electricity market data. Data in Brief, 11, 349-357. https://doi.org/10.1016/j.dib.2017.02.021

Vanfretti, L., Rabuzin, T., Baudette, M., \& Murad, M. (2016). iTesla Power Systems Library (iPSL): A Modelica library for phasor time-domain simulations. SoftwareX, 5, 84-88.

Walsh, P. P., \& Fletcher, P. (2004). Gas turbine performance. John Wiley \& Sons.

Yee, S. K., Milanovic, J. V, \& Hughes, F. M. (2008). Overview and comparative analysis of gas turbine models for system stability studies. IEEE Transactions on Power Systems, 23(1), 108-118. 